\title{
Asthma Control in Patients with Severe Eosinophilic Asthma Treated with Reslizumab: Spanish Real-Life Data
}

\author{
Luis A Pérez de Llano $\mathbb{D}^{1}, *$ \\ Borja G Cosío $\mathbb{D}^{2, *}$ \\ Ignacio Lobato Astiárraga $\mathbb{i D}^{3}$ \\ Gregorio Soto Campos $\mathbb{D}^{4}$ \\ Miguel Ángel Tejedor Alonso ${ }^{5}$ \\ Nuria Marina Malanda ${ }^{6}$ \\ Alicia Padilla Galo ${ }^{7}$ \\ Isabel Urrutia Landa ${ }^{8}$ \\ Francisco J Michel de la Rosa ${ }^{9}$ \\ Ismael García-Moguel ${ }^{10}$
}

\section{On behalf of the Reslizumab Real-Life Spanish Group}

'Pneumology Service, Hospital Lucus Augusti, Lugo, Spain; ${ }^{2}$ Pneumology Service, Hospital Universitario Son Espases-IdISBaCiberes, Palma de Mallorca, Spain; ${ }^{3}$ Pneumology Service, Complejo Asistencial de Ávila, Ávila, Spain; ${ }^{4}$ Pneumology and Allergy Unit, University Hospital of Jerez, Jerez de la Frontera, Cádiz, Spain; ${ }^{5}$ Allergy Unit, Hospital Universitario Fundación Alcorcón, Madrid, Spain; ${ }^{6}$ Pneumology Service, Hospital Universitario de Cruces, Barakaldo, Bizkaia, Spain; ${ }^{7}$ Pneumology Service, Hospital Costa del Sol, Marbella, Málaga, Spain; ${ }^{8}$ Respiratory Department, Galdakao Hospital, Galdakao, Bizkaia, Spain; ${ }^{9}$ Pneumology Service, Hospital Universitario Donostia, San Sebastian, Spain; ${ }^{10}$ Department of Allergy, Hospital Universitario 12 de Octubre, Madrid, Spain

*These authors contributed equally to this work

Correspondence: Borja G Cosío Department of Respiratory Medicine, Hospital Universitario Son ESPASESIDISBA, Palma de Mallorca, Spain Email borja.cosio@ssib.es
Background: Reslizumab is an anti-interleukin 5 monoclonal antibody that has demonstrated to reduce the risk of severe exacerbations and to improve symptoms, lung function, and quality of life in randomized controlled trials that included patients with severe eosinophilic uncontrolled asthma (SEUA) and a history of severe exacerbations.

Objective: The aim of the present study was to evaluate the effectiveness of add-on reslizumab in a cohort of patients with SEUA under real-life conditions.

Methods: This was a multi-centre, retrospective, real-life study that included subjects with SEUA treated with reslizumab in 44 asthma units throughout Spain. Eligible patients were those who had received at least one dose of reslizumab as part of normal clinical practice. The primary endpoint was complete asthma control at 52 weeks, defined as absence of severe exacerbations, ACT $\geq 20$ and no maintenance oral corticosteroids (OCS). Demographic, clinical, and functional data were collected at baseline (T0), after four to six months (T1); after 12 months (T2) and beyond 12 months of therapy (T3).

Results: Treatment with reslizumab achieved complete asthma control in $40 \%$ of the 208 included SEUA patients and led to a significant reduction in exacerbations (from 3.0; IQR: 2.0-4.0 at V0 to 0.0 ; IQR: $0.0-0.0$ at V2), maintenance OCS use (from $54.8 \%(95 \% \mathrm{CI}$ : 48.0-61.6 at T0 to $18.5 \%$ (95\% CI: $12.5-24.5$ at T2) and a meaningful improvement in symptoms in the entire treated population: ACT increased from $12.8 \pm 4.5$ at V0 to $20.0 \pm 5.1$ at V2 $(\mathrm{p}<0.001)$. Most of the improvement achieved at 12 months was obtained at $4-6$ months. The retention (continuation) rate of reslizumab was $75 \%$ through 2 years $(95 \mathrm{CI} \%$ : 1.9-2.1). Overall, reslizumab showed an adequate safety profile.

Conclusion: Reslizumab is an effective therapy for SEUA with adequate safety profile in real-life conditions.

Keywords: eosinophilic asthma, reslizumab, asthma control, monoclonal antibodies

\section{Plain Language Summary}

What is already known about this topic?

Reslizumab, an anti-interleukin 5 monoclonal antibody, reduces severe exacerbations and improves asthma symptoms, lung function, and quality of life in randomized controlled trials that included patients with severe eosinophilic uncontrolled asthma.

What does this article add to our knowledge?

Treatment with reslizumab achieved complete asthma control in $40 \%$ of these patients and led to a significant reduction in exacerbations, oral corticosteroid dose and improvement in symptoms with an adequate safety profile under real-life conditions.

How does this study impact current management guidelines? 
The study should support the inclusion of reslizumab as an effective and safe option to treat severe eosinophilic uncontrolled asthma and to aim for complete asthma control as a therapeutic goal.

\section{Introduction}

Asthma is a syndrome encompassing different phenotypes/ endotypes that imposes a growing burden on society in terms of morbidity, quality of life, and healthcare costs. It affects nearly 400 million people worldwide, ${ }^{1}$ and the prevalence of severe uncontrolled asthma (SUA) in hospital units in Spain has been estimated to be $3.9 \%{ }^{2}$

Eosinophilic asthma is a specific phenotype of asthma characterized by the presence of eosinophils in the airways. In accordance with the Global Initiative for Asthma (GINA), the eosinophilic phenotype is found in approximately $50 \%$ of severe asthma patients, ${ }^{3}$ but recently published data from The International Severe Asthma Registry (ISAR) have raised this figure up to $80 \%{ }^{4}$ Late-onset, eosinophilic asthma typically presents in the fourth or fifth decade of life and is characterized by eosinophilic bronchial inflammation that is incompletely responsive to inhaled corticosteroids (ICS). Patients often have limiting symptoms, suffer from frequent exacerbations and may be dependent on oral corticosteroids (OCS). 5,6

Significant progress has been made in the management of severe eosinophilic asthma in the past decade due to the new targeted biological therapies. These monoclonal antibodies (mAbs) may interfere at different levels of the T2 inflammatory cascade (IgE: omalizumab; IL-5: mepolizumab and reslizumab; IL-5 receptor: benralizumab and IL-4/IL-13 receptor: dupilumab) resulting in significant reduction of severe exacerbations and the need for OCS, and improvement of symptoms, quality of life and lung function. ${ }^{7}$ Nonetheless, there is a lack of validated tools to quantify the response to mAbs in severe eosinophilic uncontrolled asthma (SEUA) patients from a holistic point of view. In fact, virtually all the clinical trials have been designed to evaluate the effect of biologics on exacerbations or to assess their capacity to reduce the systemic corticosteroids' burden, but these outcomes do not cover, separately, all SEUA patients' needs. More recently, a new tool that includes all these clinically relevant variables (the FEOS score) has been developed to quantify changes from baseline. $^{8}$ Even taking into account this shortcoming, it has been reported that not all patients achieve a complete response after biologic therapy. ${ }^{9}$

Reslizumab is an IgG4-kappa humanized monoclonal antibody with a high binding affinity to IL-5. ${ }^{10}$ In Spain, the prescription of biologics for severe uncontrolled asthma shows a significant variation by geographic area, although from a global point of view, reslizumab is the least frequently prescribed anti-IL-5 biologic in our country. ${ }^{11}$ It has demonstrated to significantly reduce the risk of severe exacerbations and to improve asthma symptoms, lung function, and quality of life in two duplicate, 52-week, Phase 3, randomized controlled trials that included patients with SEUA and a history of severe exacerbations. ${ }^{12}$ Further, Wechsler et al reported that treatment with reslizumab led to improved clinical and patientreported outcomes (PRO) and significant reductions in asthma-related healthcare resource utilization (HRU) in a real-world setting. ${ }^{13}$ However, this study did not evaluate the response to reslizumab in terms of disease control and, in consequence, it does not clarify what proportion of patients achieved adequate asthma control.

Under this perspective, the aim of the present study was to evaluate the effectiveness of add-on reslizumab in a cohort of patients with SEUA under real-life conditions by using a stringent definition of response.

\section{Methods}

\section{Study Design and Patients}

This was a multi-centre, retrospective, real-life study that included adults 18 years and older with SEUA treated with reslizumab in 44 asthma units of reference throughout Spain. Eligible patients were identified by principal investigators at each asthma unit among those who had received at least one dose of reslizumab as part of normal clinical practice. The first patient was included on September $17^{\text {th }} 2020$ and the last patient was included on May $19^{\text {th }} 2021$. The drug was prescribed according to the local reimbursement requirements for reslizumab use. In Spain, reslizumab can be prescribed in patients with SUA if blood eosinophils are $\geq 400$ cells $/ \mu \mathrm{L}$ and if the patient has suffered more than two severe exacerbations (or at least two requiring hospitalization) in the preceding year, or even though the patient does not meet the exacerbation criteria, the drug can be prescribed in selected cases with limiting symptoms.

The study adhered to the Declaration of Helsinki and it was performed with approval of the local Ethics Committee of Balearic Islands (Cod 21/20; date: June $\left.5^{\text {th }} 2020\right)$. The approval was accepted by the other Autonomous Communities and every participating center signed the study contract. All patients provided written informed consent before inclusion in the study. 


\section{Objectives}

The main objective was to know the percentage of patients who achieved complete asthma control at 52 weeks, defined as absence of severe exacerbations, ACT $\geq 20$ and no maintenance oral corticosteroids (OCS).

Secondary objectives included the description of clinical and demographic characteristics of reslizumab-treated patients, asthma control at 16-24 weeks and at the latest visit (up to 30 months), changes in the number of severe exacerbations at 52 weeks, mean change from baseline in ACT score at 52 weeks, mean change from baseline in prebronchodilator FEV1 at 52 weeks, OCS burden at 52 weeks, retention rate of reslizumab (percentage of patients remaining on treatment over time) and safety of this treatment (occurrence of adverse drug reactions). Another objective was to identify predictive factors of control at 52 weeks. We have also analyzed the variation in the FEV1, Exacerbations, Oral corticosteroids, Symptoms (FEOS) score. $^{8}$ We used the FEOS score to quantify response to reslizumab. This score assigns relative weights to four domains (FEV1, exacerbations, oral corticosteroids and symptoms) covering all possible clinically relevant changes in patients' clinical condition after starting a biologic treatment. Higher scores indicate better response and the range of responses runs from 0 (worsening) to 100 (best possible response). ${ }^{8}$ The score does not provide an estimate of the level of asthma control attained after biologics therapy, but it reflects how much a given asthmatic improves. The quantification of the achieved improvement depends on the baseline disease burden, and those patients with poorer asthma control before biologic initiation have the potential to obtain higher scores after treatment than those with better pretreatment clinical condition. The worse the clinical status before treatment, the greater the room for improvement.

\section{Data Collection}

Data were collected at four different time points: "baseline" (T0, prior to treatment with reslizumab), T1, after four to six months of therapy; T2, after 12 months (10 to 14 months) of treatment and T3, the last recorded visit, beyond 12 months (up to 30 months).

For each patient we collected information concerning demographic data (age, gender, age of onset of asthma and history of smoking); atopic status; comorbidities; clinical features such as severe exacerbations (defined as deterioration in asthma resulting in unscheduled medical visit or hospitalization and oral steroids prescription for at least 3 days or increase in the maintenance dose), hospitalizations and symptoms assessed by asthma control test (ACT); ${ }^{14}$ laboratory testing (blood eosinophil counts and total serum IgE); lung function (spirometry and exhaled nitric oxide measurement: FENO); asthma medications.

\section{Statistical Analysis}

The primary effectiveness analysis was performed on the Per Protocol (PP) subject population at T2. We have collected all the information about treatment failures between visits, and all the patients who stopped treatment due to lack of efficacy or side effects were considered as non-controlled at T2 (primary effectiveness analysis) even if they did not attend the visit. The safety population comprised all patients who were included in the study and received at least one dose of reslizumab.

Statistical analysis was performed using IBM SPSS 23.0 (Armonk, NY; USA). Categorical variables were stated as numbers(n) and percentages (\%). The normality of data distribution was checked using the Shapiro-Wilk test. Results are reported as mean \pm standard deviation (SD) if normally distributed and median and interquartile range (IQR) if non-normally distributed unless indicated otherwise. Comparisons were performed using McNemar, Chi-squared or Fisher's exact test for categorical data and Student's $t$-test or Wilcoxon matched-pairs signed-rank test or Mann-Whitney- $U$-test for continuous data. p-values $<0.05$ were considered as statistically significant.

The probability of retention (continuation) of reslizumab treatment was assessed using Kaplan-Meier method. Patients were right-censored if data were not available for a specific timepoint and for patients remaining on treatment at the time of data analysis.

Two logistic regression models were created to identify which variables, measured at T0 (model 1$)$ or at $\mathrm{T} 0$ and $\mathrm{T} 1$ (model 2), can predict control at T2. In model 1, baseline variables (T0) related to control at T2 with a significance of $\mathrm{p}<0.1$ in a preliminary bivariate analysis were selected to multivariate analysis, including: age, sex, BMI $\left(<30 \mathrm{~kg} / \mathrm{m}^{2}\right.$; $\geq 30 \mathrm{~kg} / \mathrm{m}^{2}$ ), smoking (current or former), asthma onset (early or late onset; cut-off point 12 years), nasal polyposis, hospitalization in the last year (yes/no), number of exacerbations in the last year $(\leq 1 ; 2-4 ;>4)$, chronic oral corticoids use in the last year $(<5 \mathrm{mg} / \mathrm{d}$.; $\geq 5 \mathrm{mg} / \mathrm{d}$.), baseline ACT $(<14$; $\geq 14)$, and FEV1 ( $\leq 1.4 \mathrm{~L} ; 1.4 .1-2.1 \mathrm{~L} ;>2.1 \mathrm{~L})$. In model 2 , changes from $\mathrm{T} 0$ to $\mathrm{T} 1$ in ACT score (increase $<3$; $\geq 3$ ), oral corticoids (reduction; withdrawal); $\%$ exacerbation reduction $(<50 \%$; $>50-<100 \% ; 100 \%)$; FEV1 (decrease; no 
change; increase; FEV1 $\geq 80 \%$ ) were added to the set of independent variables. For both models, variables selected in the last step by forward stepwise procedure (likelihood ratio) are shown in Results.

A p value $<0.05$ was considered as statistically significant.

\section{Results}

\section{Patient Baseline Characteristics}

Two hundred and eight patients were included in the study, completing visit T0. Of these, 190 completed visit T1, 156 completed visit $\mathrm{T} 2$ and 70 completed visit $\mathrm{T} 3$. The median follow-up time for patients who reached this final visit was 94 weeks. The scheduled patients' visits during the study and the type of information collected at each study visit are shown in Figure 1.

Baseline (T0) patients' socio-demographic, clinical and functional characteristics are displayed in Table 1.

Of note, $54.8 \%$ of the patients were receiving maintenance OCS therapy, 34.1\% had been hospitalized with an asthma exacerbation and $41.8 \%$ had been treated with one or more biologics before reslizumab initiation: omalizumab, 73 patients (35.1\%); mepolizumab, 24 patients

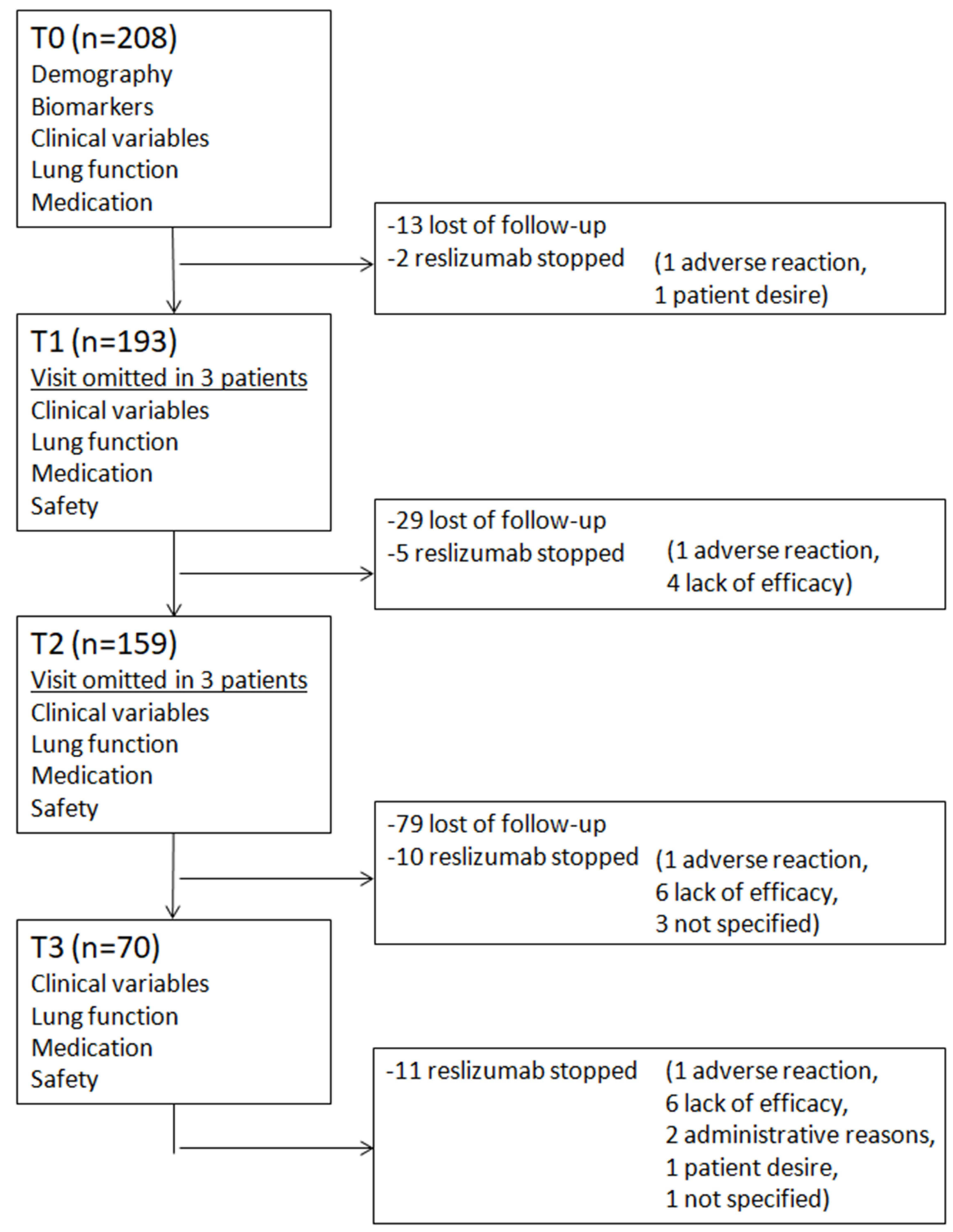

Figure I Flow chart of the study. 
Table I Population Characteristics

\begin{tabular}{|c|c|}
\hline Variable & Results \\
\hline Age (y) (mean, SD) & $56.4(11.3)$ \\
\hline Gender (\% women) & 69.7 \\
\hline BMI (mean, SD) & $27.8(5.2)$ \\
\hline Smoking history & \\
\hline- Never (\%) & 69.7 \\
\hline -Current/former (\%) & 30.3 \\
\hline -Pack-years (median, IQR) & $11.5(6.0-20.0)$ \\
\hline Onset < 12 yrs (\%) & 14.9 \\
\hline Positive skin prick-test (\%) & 43.3 \\
\hline Comorbidities (\%) & \\
\hline -Rhinitis & 52.9 \\
\hline -Sinusitis & 37.0 \\
\hline -Polyposis & 47.6 \\
\hline -NERD & 15.4 \\
\hline$-A B P A$ & 1.4 \\
\hline -Gastroesophageal reflux & 25.5 \\
\hline -Obesity & 23.1 \\
\hline -Sleep apnea & 10.1 \\
\hline -Anxiety & 22.6 \\
\hline -Depression & 15.9 \\
\hline -EGPA & 1.9 \\
\hline Blood eosinophils (cel/ $\mu \mathrm{L})$ (median, IQR) & $500.0(300.0-780.0)$ \\
\hline FENO (ppb) (median, IQR) & $33.0(13.0-58.0)$ \\
\hline FEVI (\% predicted) (mean, SD) & $70.37(23.28)$ \\
\hline FEVI/FVC (\%) (mean, SD) & $64.41(14.05)$ \\
\hline $\mathrm{FEVI} / \mathrm{FVC}<70 \%$ & 61.1 \\
\hline $\begin{array}{l}\text { Severe exacerbations in the prior } 12 \text { months (median, } \\
\text { IQR) }\end{array}$ & $3.0(2.0-4.0)$ \\
\hline Hospitalized with asthma exacerbation (\%) & 34.1 \\
\hline ICU admission & 7.9 \\
\hline $\mathrm{ACT}$ (mean, SD) & $12.84(4.49)$ \\
\hline $\mathrm{ACT}<20$ & 93.8 \\
\hline Treatment: & \\
\hline IC/LABA (\%) & 100 \\
\hline LAMA (\%) & 81.3 \\
\hline LTRA (\%) & 71.2 \\
\hline OCS maintenance therapy (\%) & 54.8 \\
\hline OCS burden (mg prednisone eq./day) (median, IQR) ${ }^{\mathrm{A}}$ & $5.6(3.0-13.5)$ \\
\hline Switched from another biologic & $41.8 \%$ \\
\hline
\end{tabular}

Notes: ${ }^{A}$ In $1 / 4$ patients who received maintenance OCS in the year before reslizumab.

Abbreviations: SD, standard deviation; ABPA, allergic bronchopulmonary aspergillosis; BMI, body mass index; EGPA, eosinophilic granulomatosis with polyangiitis; FENO, fraction of exhaled nitric oxide; FEVI, forced expiratory volume in I second FVC, forced vital capacity; IC/LABA, combination of inhaled corticosteroid and long-lasting $\beta$-agonist combination; ICU, intensive care unit; IQR, interquartile range; LAMA, long-lasting antimuscarinic agent; NERD, nonsteroidal antiinflammatory drug-exacerbated respiratory disease; LTRA, leukotriene receptor antagonist; OCS, oral corticosteroids.
(11.5\%); and benralizumab, 5 patients (2.4\%). Eleven patients $(5.3 \%)$ had previously received two biologics and 2 patients $(1 \%)$ received three biologics. Reasons for switching to reslizumab were lack of efficacy in 62 patients and side effects in 7 patients (the remaining reasons were not reported).

\section{Outcomes}

Among those patients included in the effectiveness analysis, $40 \%$ achieved control at T2 (52 weeks) according to the pre-specified definition of complete control. The percentages of patients who achieved asthma control at different time points were: $39.5 \%$ (95\% CI: 32.5-46.5) at T1; $40.0 \%$ (95\% CI: $32.3-47.8)$ at T2; and $54.3 \%$ (95\% CI: 42.3-66.2) at T2. Overall, severe exacerbations decreased from 3.0 (IQR, 2.0-4.0) at T0 to 0.0 (IQR, 0.0-0.0) at T2 $(\mathrm{p}<0.001)$. The median number of exacerbations from T0 to T3 was 0.0 (IQR, $0.0-1.0$ ). The percentage of patients under maintenance OCS decreased from $54.8 \%(95 \% \mathrm{CI}$ : $48.0-61.6)$ at T0 to $18.5 \%(95 \% \mathrm{CI}: 12.5-24.5)$ at T2 (p < $0.001)$ and the median dose of prednisone or equivalent in the 114 patients who had been receiving OCS during the year before starting reslizumab was reduced from $5.6 \mathrm{mg} /$ day (IQR, 3.0-13.5) at T0 to $0.0 \mathrm{mg} /$ day (IQR, $0.0-2.0)$ at $\mathrm{T} 2(\mathrm{p}<0.001)$. ACT increased from $12.8 \pm 4.5$ at T0 to $20.0 \pm 5.1$ at T2 $(\mathrm{p}<0.001)$. FEV1 increased from $1.85 \pm 0.73 \mathrm{~L}$ at T0 to $2.09 \pm 0.75$ at T2 $(\mathrm{p}<0.001)$. The amount of response to reslizumab, measured by the FEOS score was $74.7 \%$ (95\% CI: $71.5-78.4)$ at $\mathrm{T} 1$ and $76.4 \%$ (95\% CI: $72.5-80.3$ ) at T2, reflecting that the major clinical effect was achieved by 16 weeks with no statistically significant changes $(p=0.825)$ after 52 weeks. These results are illustrated in the Figure 2A-F.

The retention (continuation) rate of reslizumab was $75 \%$ through 2 years (95CI\%: 1.9-2.1). The cumulative reslizumab treatment period observed was 243.7 patientsyear in 193 patients. During this period 28 patients (13.5\%) withdrawn reslizumab treatment by any reason (inefficacy, 16 patients [in 4 of them with concurrent arthralgias]; adverse event, 4 patients [3arthralgias, 1 CPK elevation]; other reasons, 8 patients [2 administrative reasons; 2 patient desire; 4, not specified in clinical reports]). Probability of reslizumab treatment survival over time is plotted in Figure 3, showing a slow decrease in the first 18 months. Cumulative reslizumab survival at months $6,12,18$ and 24 was $95.1 \%, 92.0 \%, 85.1 \%$ and $73.4 \%$ respectively. 
A

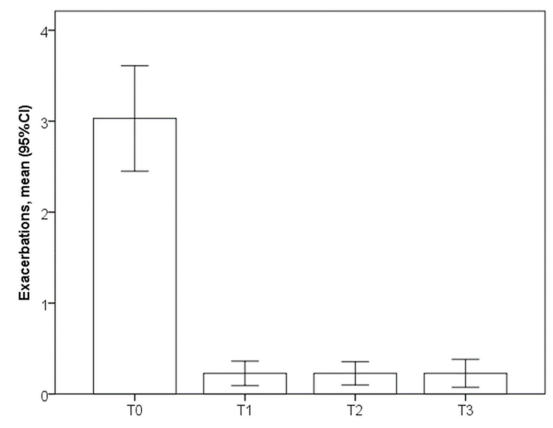

D

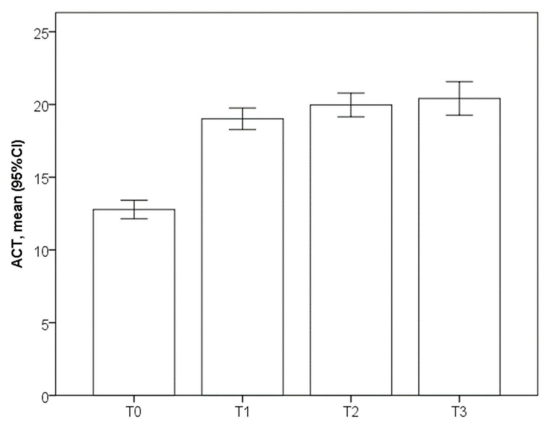

B

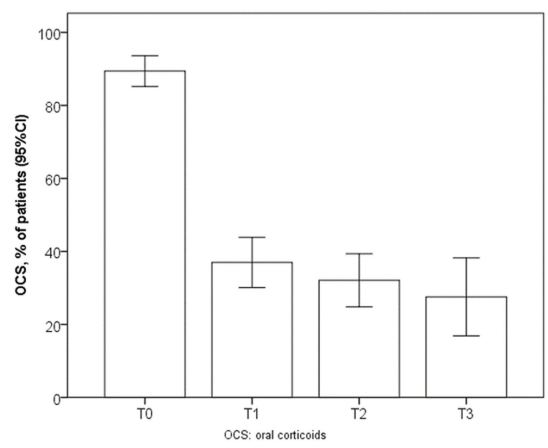

E

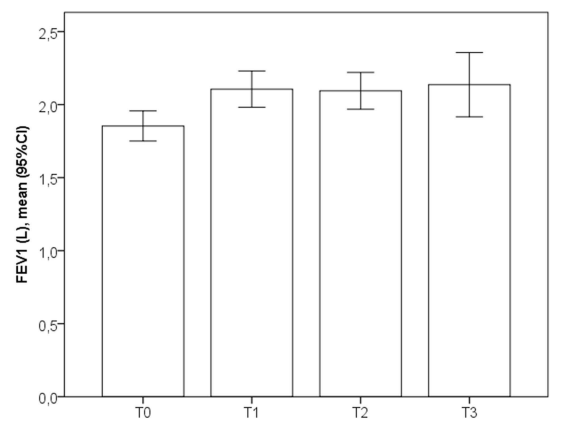

C

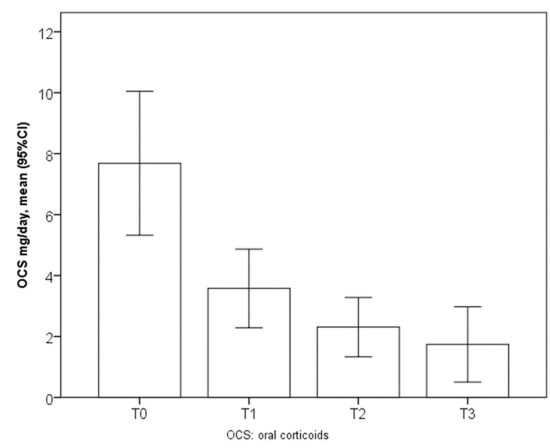

$\mathbf{F}$

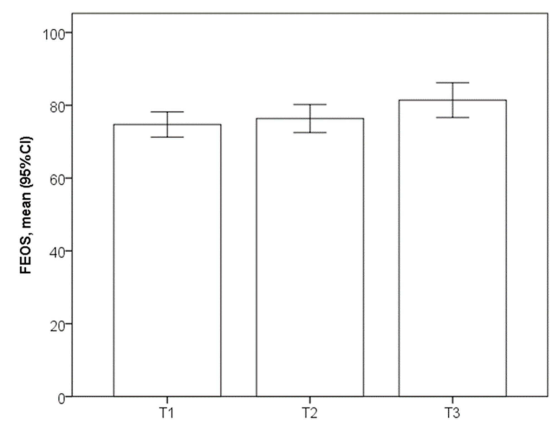

Figure 2 This figure illustrates the changes in the outcome variables across the different visits (A) severe exacerbations; (B) percentage of patients with maintenance oral corticosteroids (OCS); (C) dose of maintenance OCS, mg/day prednisone equivalent; (D) asthma control test; (E) forced expiratory volume in I second (FEVI); (F) FEOS score. Results are shown as mean and $95 \%$ confidence interval $(95 \% \mathrm{Cl})$.

\section{Factors Predicting the Achievement of Control at 52 Weeks}

In a multivariate analysis, ACT <14 (OR 3.471; IC95\%: 1.376-8.757) at T0 was found to be an independent factor associated to asthma control (according to definition 1) at T2. Corticosteroids' withdrawal (OR 9.243; IC95\%: 4.064-21.023) and an ACT increase $\geq 3$ (OR 1.101; IC95\%: 1.016-1.194) at T1 were significantly associated with asthma control at $\mathrm{T} 2$.

\section{Safety Profile}

Among the 28 patients (13.5\% of the whole sample) who discontinued reslizumab, four of them discontinued due to adverse events (arthromyalgias in three cases and elevated Creatine Kinase levels in one). Adverse events occurred in 20 patients $(9.6 \%)$. The most frequently reported ones were arthromyalgia (5.3\%) followed by headache $(1.9 \%)$.

\section{Discussion}

We carried out the first real-world study of reslizumab in patients with uncontrolled severe eosinophilic asthma in Spain. The main finding is that reslizumab was able to achieve complete asthma control in $40 \%$ of the patients at 52 weeks in accordance with the pre-specified definition (no exacerbations, controlled symptoms and no maintenance OCS). Overall, our patients experienced a significant reduction in the number of severe exacerbations and OCS dose, with a concomitant improvement in asthma symptoms and lung function. Although our population was composed of particularly severe asthma patients $(54.8 \%$ were receiving maintenance OCS therapy, $34.1 \%$ had been hospitalized with an asthma exacerbation and $43 \%$ had been treated with another biologic before reslizumab initiation), the observed results are in line with those reported in reslizumab controlled trials ${ }^{12}$ and subsequent published real-world studies. ${ }^{13,15}$ Wechsler et al observed an excellent response in $58.6 \%$ of reslizumabtreated patients, ${ }^{13}$ but being response restricted to asthma exacerbations, without taking into consideration other essential outcomes such as symptoms and OCS use. In this regard, we think that the aim of a biological therapy should be complete asthma control and therefore, the response to biologics should be evaluated from a holistic point of view bringing together all the relevant therapeutic goals. It has been published that "partial" response is the 


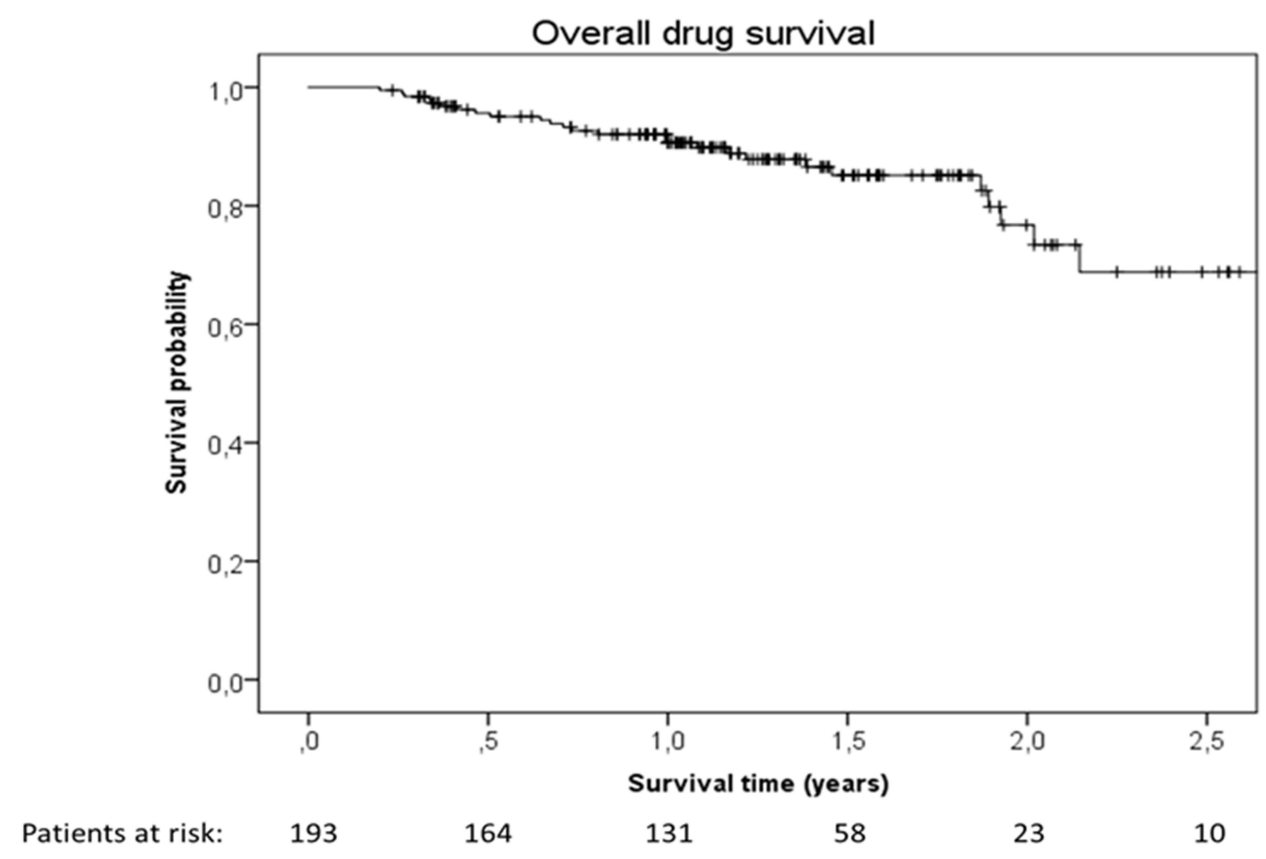

Figure 3 Overall drug survival curve.

most frequently found one in biologic-treated SUA patients. ${ }^{9}$ Since many reports showed benefit for switching to other biologics, ${ }^{16,17}$ clinicians have to be demanding about the quality and quantity of the response. In this study, we have adopted a stringent definition of response, a definition that resembles the so-called "clinical remission" concept proposed by a group of influential authors in an attempt to push forward asthma treatment and improve long term outcome, similar to what has been accomplished in other chronic inflammatory diseases such as rheumatoid arthritis. $^{18}$

Response assessment by the FEOS score was $74.7 \%$ at $\mathrm{T} 1$ and $76.4 \%$ at $\mathrm{T} 2$, reflecting that the major clinical effect was achieved within 16 weeks of reslizumab initiation, with modest further improvement at 52 weeks. This finding is in accordance with those of previous studies with other anti-IL5 mAbs: data from two mepolizumab clinical trials (COSMO and COSMEX) clearly show that most of the response in symptoms, lung function, and even oral corticosteroids reduction is achieved at 16 to 24 weeks after biologic start. ${ }^{19,20}$ The same applies to benralizumab, which appears to give the most of its benefits after 4-16 weeks of treatment. ${ }^{21,22}$ Therefore, if symptoms and FEV1 do not improve at 24 weeks and corticosteroids' dose cannot be tapered down at this time point, a complete response cannot be expected later on. Interestingly, 12 patients stopped reslizumab after one year of treatment, reflecting that more clear and specific recommendations about how to measure and interpret response to biologics are needed. Of note, $42 \%$ of our patients had failed to respond to other biologics. This is in keeping with our previous results showing that Reslizumab is an effective and safe option for patients with severe eosinophilic asthma and a history of omalizumab failure. ${ }^{17}$

The retention rate of treatment with biological therapy, or the probability of maintaining the treatment over time, is a good criterion for evaluating the efficacy/tolerance balance in a real-world setting. ${ }^{23}$ In this study, the retention rate of reslizumab was $75 \%$ through 2 years. The fact that reslizumab was interrupted because of lack of efficacy in 16 patients -a figure similar to that of other real-life published studies ${ }^{9}$ - might have different explanations: wrong identification of the underlying inflammatory profile (T2-high inflammation is heterogeneous, even in patients who share the same clinical phenotype), the coexistence of conditions such as emphysema that may negatively impact the possibility of improvement $(30 \%$ of the patients were current or ex-smokers), because insufficient dose (unlikely in the case of a weight-dosed drug) or because the subsequent development of infections. On the other hand, reslizumab showed a favorable safety profile. Only 20 out of 208 patients (9.6\%) experienced 
adverse events, with four cases leading to treatment withdrawal.

At baseline (T0), a high symptoms burden $(\mathrm{ACT}<14)$ was found to be an independent risk factor influencing asthma control at T2 (maybe pointing at the coexistence of COPD). Corticosteroids' withdrawal and achievement of the minimal clinically important difference in ACT at $\mathrm{T} 1$ were significantly associated with asthma control at $\mathrm{T} 2$ (something that seems logical, since both symptoms and OCS use are markers of early response).

This study has several strengths, including the multicentric character of the research, the relatively large size (208 patients) of the population with particularly severe disease, the "before and after" design that reflects the routine clinical practice in a real-world setting, the long duration of follow-up (median 94 weeks for those patients who reached the final visit) and the choice of a composite outcome measure based on clinically relevant variables. The major drawback of our study relies on its retrospective and non-randomized design, which may have led to incomplete information or biases. In a retrospective study like this, per protocol analysis is subject to selection bias due to loss to follow up. Since per protocol refers to inclusion in the analysis of only those patients who strictly adhered to the protocol (treatment and scheduled visits), the proportion of responders among those who complete treatment may provide an exaggerated estimate of treatment effect. However, in an effort to tackle this problem, we have collected all the information about treatment failures and all the patients who stopped treatment due to lack of efficacy or side effects were considered as noncontrolled at T2 (primary effectiveness analysis), even if they did not attend the visit.

In conclusion, this real-life study indicates that treatment with reslizumab achieves complete asthma control in around half of the included severe asthma patients. This therapy also led to a significant reduction in exacerbations, OCS dose and a meaningful improvement in symptoms. Most of the improvement achieved at 12 months was obtained at 4-6 months. Overall, reslizumab showed an adequate safety profile. These results complement those of reslizumab pivotal studies.

\section{Acknowledgments}

Authors would like to thank Dr Marta Villarnovo and BRINDA Healthcare for their assistance with the logistics of data collection and analysis. The full list of the Reslizumab real-life Spanish group is included in the Appendix.

\section{Authors' Contributions to the Study}

Luis A Pérez de Llano, Borja G Cosío, Ignacio Lobato Astiárraga, Gregorio Soto Campos, Miguel Ángel Tejedor Alonso, Nuria Marina Malanda, Alicia Padilla Galo, Isabel Urrutia Landa, Francisco J Michel de la Rosa, Ismael García-Moguel made a significant contribution to the work reported, in the conception, study design, execution, acquisition of data, analysis and interpretation; have drafted or written, or substantially revised or critically reviewed the article, have agreed on the journal to which the article will be submitted; reviewed and agreed on all versions of the article before submission, during revision, the final version accepted for publication, and any significant changes introduced at the proofing stage; agree to take responsibility and be accountable for the contents of the article.

\section{Funding}

This project was supported by the integrated asthma research program of the Spanish Respiratory Society (PII de asma SEPAR), with no role in the analysis, decision to publish or preparation of the manuscript. The design, analysis, and writing of this report are entirely the work and responsibility of the authors, and Dr. Cosío and Dr Pérez de Llano had full access to all data and final responsibility for the decision to submit this work for publication.

\section{Disclosure}

Luis A Pérez de Llano declares to have received grants and/or fees for consultancy or speeches from Novartis, Astra-Zeneca, GSK, Teva, Boehringer-Ingelheim, Chiesi, Sanofi, MSD, FAES, Techdow Pharma, Leo Pharma, Gebro, Gilead, Menarini, Mundipharma, and Esteve. Borja G Cosío declares he has received speaking or advisory fees, or economic aid to attend congresses from Astra-Zeneca, GSK, Novartis, Chiesi, Mundipharma, Menarini, Sanofi, TEVA, Boehringer-Ingelheim, and Rovi. Ignacio Lobato Astiárraga declares he has received speaker fees, consulting fees from Astra-Zeneca, GlaxoSmithKline, Novartis, Chiesi, Sanofi, Boehringer, SEPAR, and Teva. Gregorio Soto Campos declares he has received speaker fees, consulting fees from ALK, Astra-Zeneca, Bial, Boehringer-Ingelheim, Chiesi, GlaxoSmithKline, Novartis, Sanofi, and Teva. Miguel Ángel Tejedor Alonso declares that there is no relevant conflict of interest. Nuria Marina Malanda declares she has 
received speaker fees, consulting fees from Astra-Zeneca, Sanofi, GlaxoSmithKline, Chiesi, Pfizer, Novartis, and Teva. Alicia Padilla Galo declares she has received speaking or advisory fees, or economic aid to attend congresses from Novartis, Teva, Astra-Zeneca, GlaxoSmithKline, and ALK. Isabel Urrutia Landa declares she has received speaking or advisory fees, or economic aid to attend congresses from Astra-Zeneca, Sanofi, GlaxoSmithKline, Chiesi, Bial Aristegui, Teva, Novartis, ALK, BoehringerIngelheim, and Mundi Pharma. Francisco Javier Michel de la Rosa has received speaker fees and/or consulting fees and/or support attend Congresses from the following: Astra-Zeneca, Boehringer-Ingelheim, Chiesi, CSL Behring, GlaxoSmithKline, Grifols, Menarini, Novartis, Sanofi Aventis, and Teva. Ismael García-Moguel declares he has received speaking or advisory fees, or economic aid to attend congresses from Astra-Zeneca, Sanofi, GlaxoSmithKline, Chiesi, Mundipharma, Allergy therapeutics, Novartis, Stallergenes Greer, and Teva. The authors report no other conflicts of interest in this work.

\section{References}

1. GBD 2016 Disease and Injury Incidence and Prevalence Collaborators. Global Burden of Disease 2016 Disease and Injury Incidence and Prevalence Collaborators. Global, regional, and national incidence, prevalence, and years lived with disability for 328 diseases and injuries for 195 countries, 1990-2016: a systematic analysis for the Global Burden of Disease Study 2016. Lancet. 2017;390:1211-1259. doi:10.1016/S0140-6736(17)32154-2

2. Quirce S, Plaza V, Picado C, Vennera M, Casafont J. Prevalence of uncontrolled severe persistent asthma in pneumology and allergy hospital units in Spain. J Investig Allergol Clin Immunol. 2011;21 (6):466-471.

3. Global Initiative for Asthma. Global strategy for asthma management and prevention; 2021. Global Initiative for Asthma website. Available from: https://ginasthma.org/wp-content/uploads/2021/05/GINA-MainReport-2021-V2-WMS.pdf. Accessed December 30, 2021.

4. Heaney LG, Perez de Llano L, Al-Ahmad M, et al. Eosinophilic and noneosinophilic asthma: an expert consensus framework to characterize phenotypes in a global real-life severe asthma cohort. Chest. 2021;160(3):814-830. doi:10.1016/j.chest.2021.04.013.

5. Amelink M, de Groot JC, de Nijs SB, et al. Severe adult-onset asthma: a distinct phenotype. J Allergy Clin Immunol. 2013;132:336-341. doi:10.1016/j.jaci.2013.04.052

6. de Groot JC, Storm H, Amelink M, et al. Clinical profile of patients with adult-onset eosinophilic asthma. ERJ Open Res. 2016;2:2015. doi: $10.1183 / 23120541.00100-2015$

7. Agache I, Akdis CA, Akdis M, et al. EAACI Biologicals Guidelines-Recommendations for severe asthma. Allergy. 2021;76 (1):14-44. doi:10.1111/all.14425

8. Pérez de Llano L, Dávila I, Martínez-Moragón E, et al. Development of a Tool to Measure the Clinical Response to Biologic Therapy in Uncontrolled Severe Asthma: the FEV1, Exacerbations, Oral Corticosteroids, Symptoms Score. J Allergy Clin Immunol Pract. 2021;9(7):2725-2731. doi:10.1016/j.jaip.2021.01.033.
9. Eger K, Kroes JA, Ten Brinke A, Bel EH. Long-Term Therapy Response to Anti-IL-5 Biologics in Severe Asthma-A Real-Life Evaluation. J Allergy Clin Immunol Pract. 2021;9(3):1194-1200. doi:10.1016/j.jaip.2020.10.010

10. Liddament M, Husten J, Estephan T, et al. Higher Binding Affinity and in vitro Potency of Reslizumab for Interleukin-5 Compared With Mepolizumab. Allergy Asthma Immunol Res. 2019;11(2):291-298. doi:10.4168/aair.2019.11.2.291

11. Casas-Maldonado F, Álvarez-gutiérrez FJ, Blanco-Aparicio M, et al. Monoclonal antibody treatment for severe uncontrolled asthma in Spain: analytical map. $J$ Asthma. 2021;17:1-11. doi:10.1080/ 02770903.2021.1978483

12. Castro M, Zangrilli J, Wechsler ME, et al. Reslizumab for inadequately controlled asthma with elevated blood eosinophil counts: results from two multicenter, parallel, double-blind, randomized, placebo-controlled, phase 3 trials. Lancet Respir Med. 2015;3 (5):355-366. doi:10.1016/S2213-2600(15)00042-9

13. Wechsler ME, Peters SP, Hill TD, et al. Clinical outcomes and health-care resource use associated with reslizumab treatment in adults with severe eosinophilic asthma in real-world practice. Chest. 2021;159(5):1734-1746. doi:10.1016/j.chest.2020.11.060

14. Crimi C, Campisi R, Noto A, et al. Comparability of asthma control test scores between self and physician-administered test. Respir Med. 2020;170:106015. doi:10.1016/j.rmed.2020.106015

15. Ibrahim H, O'Sullivan R, Casey D, et al. The effectiveness of Reslizumab in severe asthma treatment: a real-world experience. Respir Res. 2019;20(1):289. doi:10.1186/s12931-019-1251-3

16. Numata T, Araya J, Miyagawa H, et al. Effectiveness of Switching Biologics for Severe Asthma Patients in Japan: a Single-Center Retrospective Study. $J$ Asthma Allergy. 2021;14:609-618. doi:10.2147/JAA.S311975

17. Pérez de Llano LA, Cosío BG, Domingo C, et al. Efficacy and Safety of Reslizumab in Patients with Severe Asthma with Inadequate Response to Omalizumab: a Multicenter, Open-Label Pilot Study. J Allergy Clin Immunol Pract. 2019;7(7):2277-2283. doi:10.1016/j. jaip.2019.01.017

18. Menzies-Gow A, Bafadhel M, Busse WW, et al. An expert consensus framework for asthma remission as a treatment goal. J Allergy Clin Immunol. 2020;145(3):757-765. doi:10.1016/j.jaci.2019.12.006

19. Lugogo N, Domingo C, Chanez $P$, et al. Long-term efficacy and safety of mepolizumab in patients with severe eosinophilic asthma: a multi-center, open-label, phase IIIb study. Clin Ther. 2016;38 (9):2058-2070. doi:10.1016/j.clinthera.2016.07.010

20. Khurana S, Brusselle GG, Bel EH, et al. Long-term safety and clinical benefit of mepolizumab in patients with the most severe eosinophilic asthma: the COSMEX study. Clin Ther. 2019;41 (10):2041-2056. doi:10.1016/j.clinthera.2019.07.007

21. Pelaia C, Crimi C, Benfante A, et al. Therapeutic Effects of Benralizumab Assessed in Patients with Severe Eosinophilic Asthma: real-Life Evaluation Correlated with Allergic and Non-Allergic Phenotype Expression. $J$ Asthma Allergy. 2021;14:163-173. doi:10.2147/JAA.S297273

22. Nolasco S, Crimi C, Pelaia C, et al. Benralizumab Effectiveness in Severe Eosinophilic Asthma with and without Chronic Rhinosinusitis with Nasal Polyps: a Real-World Multicenter Study. J Allergy Clin Immunol Pract. 2021;9(12):4371-4380.e4. doi:10.1016/j. jaip.2021.08.004

23. Blum MA, Koo D, Doshi JA. Measurement and rates of persistence with and adherence to biologics for rheumatoid arthritis: a systematic review. Clin Ther. 2011;33(7):901-913. doi:10.1016/j. clinthera.2011.06.001 


\section{Publish your work in this journal}

The Journal of Asthma and Allergy is an international, peer-reviewed open-access journal publishing original research, reports, editorials and commentaries on the following topics: Asthma; Pulmonary physiology; Asthma related clinical health; Clinical immunology and the immunological basis of disease; Pharmacological interventions and

Submit your manuscript here: https://www.dovepress.com/journal-of-asthma-and-allergy-journal new therapies. The manuscript management system is completely online and includes a very quick and fair peer-review system, which is all easy to use. Visit http://www.dovepress.com/testimonials.php to read real quotes from published authors. 